\title{
CYTOSKELETAL F-ACTIN PATTERNS IN SKIN FIBROBLASTS FROM NORMAL SUBJECTS AND PATIENTS WITH TUBEROUS SCLEROSIS AND VON RECKLINGHAUSEN'S NEUROFIBROMATOSIS
}

\author{
Kousaku Ohno, Sachio Takashima, and Kenzo Takeshita \\ Division of Child Neurology, Institute of Neurological Sciences, \\ Tottori University School of Medicine, Yonago 683, Japan
}

\begin{abstract}
Summary Cytoskeletal F-actin of formaldehide fixed, acetone-extracted cells was stained with 7-nitrobenz-2-oxa-1,3-diazole(NBD)-phallacidin. Skin fibroblasts from apparently normal fetuses, children and adults, and from patients with tuberous sclerosis (TS) (6 strains) and von Recklinghausen's neurofibromatosis (NF) (4 strains) were examined. Skin fibroblasts from patients with adenomatosis of the colon and rectum (ACR) and basal cell naevus syndrome (BCN) and simian virus 40 (SV40) transformed skin fibroblasts were also included. About $75 \%$ of the SV 40 transformed cells had less or no organized actin fibers, as repeatedly reported for many virally transformed cell systems. In rapidly proliferating cultures of fibroblasts from normal subjects, highly organized action fibers were seen in 74.8 and $77.3 \%$ of the cells from fetal and child cell cultures, respectively, and in $84.0 \%$ of cells from adult cell cultures at the same low levels of in vitro passage $(\mathrm{p}<0.001)$. This suggests that the organization of actin fibers increases with age and that the change is similar to that during in vitro senescence. In fibroblasts from patients with $\mathrm{TS}$ and NF, the organization of actin fibers was almost the same as that of normal fibroblasts.
\end{abstract}

\section{INTRODUCTION}

Actin has been found in eukaryotic non-muscle cells and is related to a wide variety of cellular functions. The organization of the actin bundles changes after transformation with many oncogenic viruses and during in vitro senescence. In general, the actin bundles in virus-transformed cells are decreased in size and number, which is considered to be one of the major correlates of in vitro transformation (Weber et al., 1974; Verderame et al., 1980). Increased organization of the actin bundles during in vitro senescence is frequently correlated with enlargement of cell

Received April 1, 1985; revised version received September 30, 1985 
size and is suggested to be related to the inability of the senescent cells to replicate (Wang and Gundersen, 1984).

The risk of developing some tumors has been recognized for many years to be a familial trait. There are neoplasms which follow a pattern of autosomal dominant inheritance. In familial adenomatosis of the colon and rectum (ACR), cultured skin fibroblasts have many properties observed for transformed cells (for a review, see Kopelovitch, 1982). In retinoblastoma and von Recklinghausen's neurofibromatosis (NF), other diseases with autosomal dominant mutations predisposed to neoplasia, skin fibroblasts have been reported to have some similarities to transformed cells: enhanced growth in a suboptimal concentration of fetal calf serum (Kossakowska et al., 1982; Krone et al., 1981). These phenomena are important for the understanding of a genetic background prone to the development of neoplasia and for the clarification of the pathogenesis of several autosomal dominant disorders predisposed to neoplasia.

To determine whether the actin organization of cells from patients with tuberous sclerosis (TS) and von Recklinghausen's neurofibromatosis (NF), which are autosomal dominant disorders prone to the development of neoplasia (Mulvihill, 1977), has any similarity to transformed cells, we studied the F-actin organization in skin fibroblasts from normal individuals and from patients with these and other disorders.

\section{MATERIALS AND METHODS}

Cells and culture conditions. Skin biopsies were performed on apparently normal subjects including four fetuses (17 to 38 weeks of gestation), nine children ( 2 months to 8 years of age) and 11 adults (18 to 76 years of age), and patients including six with TS $(1,14,16,26,31$ and 33 years old), four with NF $(6,10,12$ and 40 years old), and one with basal cell naevus syndrome (BCN, 10 years old). Fibroblast cultures were initiated from the skin biopsies by the method described previously (Ohno and Takeshita, 1984a). Two ACR strains (from 40- and 58-year-old patients) and one ACR strain (from a 37-year-old patient) were obtained from Dr. E. Kaneto, National Kure Hospital, and Dr. N. Kaibara, Department of Surgery, Tottori University Hospital, respectively. A simian virus 40 (SV40) transformed cell line (SVLN1) was established from SV40 infected cultures of skin fibroblasts from a patient with Lesch-Nyhan syndrome as described previously (Ohno and Kimura, 1984). Cells were grown and maintained in Dulbecco's modified Eagle's medium (DEM) supplemented with $10 \%$ fetal bovine serum (FBS). Cultures showing population doubling levels between 8 and 12 were used for the experiments.

Determination of F-actin distribution in skin fibroblasts. Exponentially proliferating cells were seeded at an initial plating density of $1 \times 10^{4}$ cells per $3.5 \mathrm{~cm}$ dish with a $1.8 \mathrm{~cm} \times 1.8 \mathrm{~cm}$ glass coverslip in DEM supplemented with $10 \%$ FBS. Three days after subculturing, cells were rinsed with phosphate-buffered saline (PBS) and were fixed in a $3.7 \%$ formaldehyde solution in PBS without $\mathrm{Ca}^{2+}$ and $\mathrm{Mg}^{2+}$ 
for $10 \mathrm{~min}$ at room temperature. Staining with 7-nitrobenz-2-oxa-1,3-diazole(NBD)phallacidin (Molecular Probes, Texas) was performed by the method of Barak et al. (1981) after extraction with a solution of $-20^{\circ} \mathrm{C}$ acetone. Fixed and extracted coverslips were examined at various times during the day with an Olympus fluorescence microscope (Model BH-2-RFL, Olympus Optical Co., Ltd.).

Categories of F-actin organization. Cytoskeletal F-actin patterns of individual cells were divided into the following categories by modifying the criteria of Verderame et al. (1980): class 1 cells have at least one heavy distinct fiber running along the long axis of the cytoplasm (Fig. 1, A and B), class 2 cells have no long distinct fibers but have fine and short fibers that enter the central half of the cells (Fig. 1, C and D), and class 3 cells have a few fine cables only in the peripheral area of the cells (Fig. 1, $\mathrm{E}$ and $\mathrm{F}$ ). Rounded cells in the mitotic phase, a few percent of the total cells, were included in class 3. More than 250 cells on each coverslip from 3 independent dishes were scored for each strain.

\section{RESULTS}

Using an indirect immunofluorescent method, Kopelovitch et al. (1977, 1980) have quantitated the actin patterns of cells cultured in a medium containing $1 \%$ FBS for $48 \mathrm{hr}$ and kept in $3.8 \%$ formaldehyde for four to eight days at $4{ }^{\circ} \mathrm{C}$ before acetone extraction and staining. When we cultured cells derived from healthy donors in DEM containing $1 \%$ FBS for $48 \mathrm{hr}$, the cells ceased to proliferate and increase in size, and almost $100 \%$ of the cells contained numerous prominent actin cables traversing the entire long axis of the cytoplasm (class 1) as can be seen in Fig. $1 \mathrm{~A}$. Whereas SV40 transformed SVLN1 cells continued to proliferate in DEM containing $1 \%$ FBS with slower population doubling time and reached almost the same saturation density, when compared to the proliferation in DEM containing $10 \%$ FBS (data not shown). The different proliferative potentials in medium containing low serum may alter the proportions of cells containing each category of actin fibers. To eliminate this possibility, we compared the organization of actin in exponentially proliferating cultures in medium containing $10 \%$ FBS.

The distribution of actin cables in proliferating SV40 transformed SVLN1 cells was reproducibly different from that in proliferating cultures from normal individuals of various ages in medium containing $10 \%$ FBS. The SVLN1 cultures contained many cells with fine fibers (classes 2 and 3, Fig. 1, C and E), but a small number of cells had large fibers of class 1 (Fig. 2).

In the histogram for each strain from different aged donors, the percentage of class 1 cells showed a gradual increase with the aging of the donors (Fig. 2). Furthermore, in a series of skin fibroblasts from normal individuals of various ages, cultures from adult donors contained significantly more class 1 cells than the level in cultures from child donors (Fig. 3).

Figure 4 shows the proportion of class 1 cells in skin fibroblast strains in several 

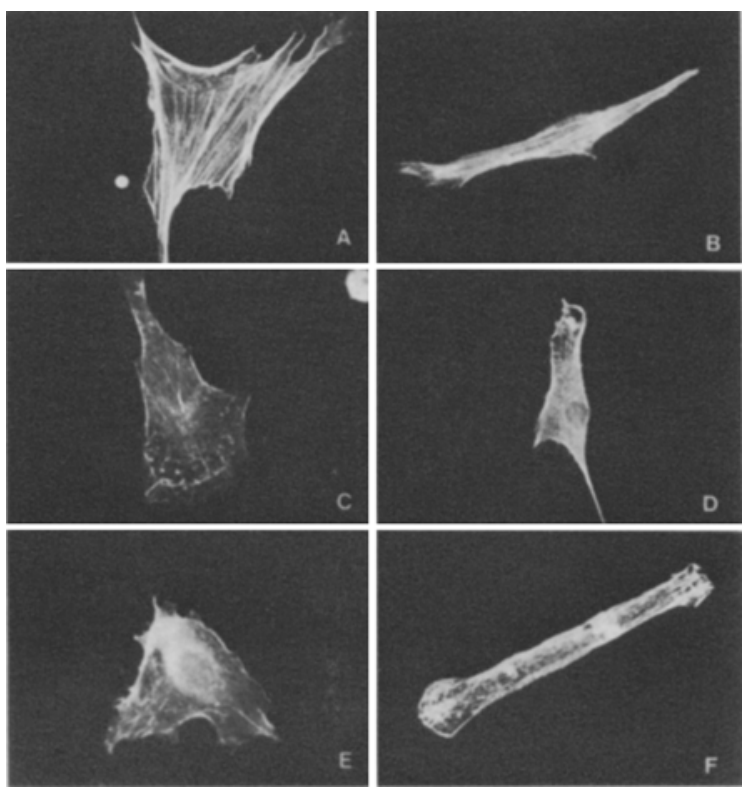

Fig. 1. Three categories of F-actin distribution used for scoring cultures stained with NBD-phallacidin. (A) and (B) are class 1, (C) and (D) are class 2, and (E) and (F) are class 3, as described in Materials and Methods. The cell shown in (A) was a cell from a normal subject cultured in DEM containing $1 \% \mathrm{FBS}$. The three cells in (B), (D) and (F) were cells from normal subjects and the two cells in (C) and (E) were SV40 transformed cells in the rapidly proliferating phase in DEM containing $10 \%$ FBS.
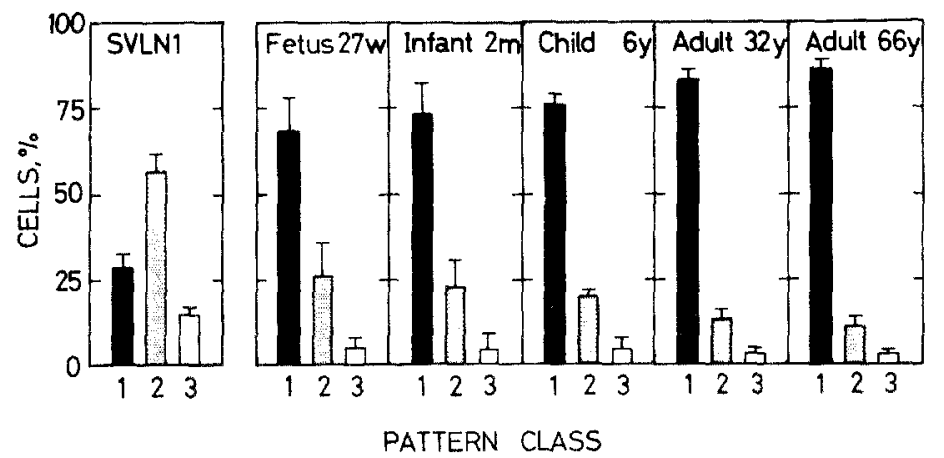

Fig. 2. F-actin pattern distribution in SV40 transformed (SVLN1) cells and cells from normal individuals of various ages. For each strain, more than 250 cells were scored on at least three coverslips each. The results are means $=\mathrm{SD}$ for each score of each category. 


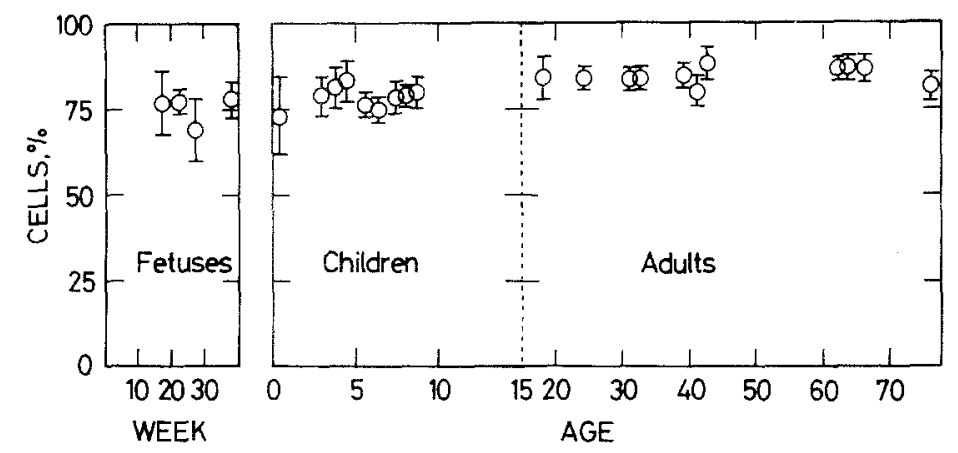

Fig. 3. Percentages of cells (mean $\pm S D$ ) containing actin fibers traversing the entire long axis of the cytoplasm (class 1 ) in cultures from normal individuals of various ages. The mean values ( $\pm \mathrm{SD}$ ) for fetal, child and adult groups were $74.8 \pm 3.6,77.3 \pm 4.0$ $(0.1<\mathrm{p}<0.2$, from the level of the fetal group), and $84.7 \pm 2.4$ ( $\mathrm{p}<0.001$, from the level of the child group), respectively.

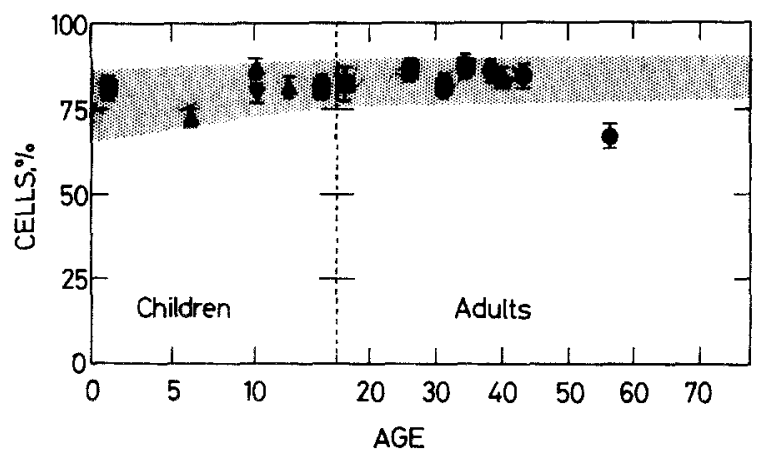

Fig. 4. Percentages of cells (mean $\mathrm{SD}$ ) containing class 1 actin cables in cells from patients

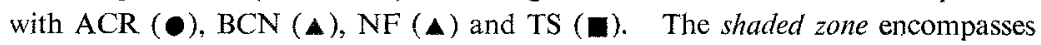
control zone.

autosomal dominant disorders. Except for one strain from a patient with ACR, all cultures from two ACR, one BCN, four NF and six TS patients showed the normal range of class 1 cells compared with the data for normal individuals of various ages. The strain from a 58-year-old ACR patient contained about $70 \%$ of class 1 cells, corresponding to the level in fetal cultures.

\section{DISCUSSION}

Among several autosomal dominant disorders predisposed to neoplasia, cultured skin fibroblasts from ACR patients have been reported to show not only increased sensitivity to transformation by oncogenic viruses (Pfeffer and Kopelovitch, 1977; 
Rasheed et al., 1983) and chemical agents (Kopelovitch et al., 1979; Rhim et al., 1980), but also similarities to transformed cells, in enhanced growth in medium containing a low concentration of serum (Pfeffer et al., 1976), loss of contact inhibition of proliferation (Kopelovitch, 1977) and defective actin-containing cables (Kopelovitch et al., 1977, 1980). Based on their extensive data, Kopelovitch (1982) contends that the transformed phenotype in vitro, which is equal to cancer predisposition in vivo, is due to an autosomal dominant mutation for most forms of hereditary cancer syndromes and for sporadic forms of cancer. According to this hypothesis, Azzarone et al. (1984) found that skin fibroblasts from patients with breast cancer behaved in vitro like transformed cells with respect to in vitro life span, anchorage independent growth and saturation density. Although skin fibroblasts from patients with retinoblastoma (Kossakowska et al., 1982) and NF (Krone et al., 1981) have also been reported to multiply better than control cells in suboptimal serum conditions, a conclusion that the cells from these disorders have definite enhanced proliferative potentials was not drawn, because of significant variability of proliferative potentials from strain to strain. We (Ohno and Takeshita, 1984b) observed that some of TS and NF strains reached higher cell densities than control cells in a suboptimal concentration of medium, but the ability of TS and NF cells to proliferate in that condition was obviously less than that of SV 40 transformed cells.

The methods we used to reveal cytoskeletal F-actin were different from those of Verderame et al. (1980) and Kopelovitch et al. (1977, 1980), but we could easily differentiate the SV40 transformed human skin fibroblast culture from many fibroblast cultures derived from apparently normal individuals of various ages. Under these conditions, we could not find any abnormal patterns of F-actin distribution of cells from NF, TS and BCN patients. This shows that skin fibroblasts from NF and TS patients have no similarities to virally transformed fibroblasts. Neoplasms in the skin and the nervous system are frequently associated with NF and TS, and the malignant transformation of such neoplasms is also known. However, neurofibroma and sarcomatous degeneration of neurofibroma in NF, and angiofibroma, glioma and malignant glioma in TS usually originate in non-epithelial tissues. The disorders in which fibroblasts are known to show similarities to transformed cells in some growth characteristics are predisposed to neoplasms of the epithelial origin. These disorders include polyposis and cancer of the colon and the rectum, and breast cancer. This may be one of reasons why fibroblasts from NF and TS patients do not show any similarities to transformed cells with respect to proliferative potentials and F-actin organization.

ACR fibroblasts cultured in medium supplemented with $1 \%$ serum have been reported to show abnormal F-actin patterns (Kopelovitch et al., 1977, 1980). Under this nutrient condition, ACR fibroblasts are also reported to proliferate better than control fibroblasts (Pfeffer et al., 1976). Different F-actin patterns observed in a suboptimal concentration of serum may result from enhanced proliferative potentials of ACR fibroblasts. The results described here show that in exponentially 
proliferating cultures in medium with $10 \%$ serum, one of three ACR cultures contained a high percentage of less-organized actin cables. This may partly support the results of Kopelovitch et al. $(1977,1980)$. However, in proliferating cultures in $10 \%$ serum medium, we could not observe abnormal F-actin organization in cells from two of three ACR patients.

Several parameters that change with increased in vitro cell aging change as a function of age in vivo. Total replicating capacity (in vitro life span), cell population replication rate, and colony size distribution are found to decrease in both late passage cultures (in vitro cellular aging) and cultures from older donors (in vivo cellular aging) (Schneider and Mitsui, 1976; Smith et al., 1978). Recently, the decline of replicative potentials during in vitro cellular aging is correlated with increased organization of the cytoskeleton (Wang and Gundersen, 1984). Our study shows that the actin organization observed in fetal, child and adult human cell cultures increases with the age of the donor. This phenomenon resembles the increase in actin organization during in vitro senescence (Wang and Gundersen, 1984), but the physiologic significance is unknown. Cultures from aged donors show an increase in both cell population replication time and the percentage of nonreplicating cells (Schneider and Mitsui, 1976). The majority of slowly replicating or nonreplicating cells in senescent cultures accumulate in the Gl phase of the cell cycle (Yanishevsky et al., 1974). This suggests that cultures from older donors contain more cells in the G1 (G0) phase of the cell cycle than cultures from younger donors. Increased actin organization in cultures from older donors may be correlated with the increased number of G1 (G0) cells in cultures. In fact, as shown in our preliminary experiment, most cells cultured in a medium containing $1 \%$ FBS, which usually accumulate in a state of quiescence in the Gl (G0) phase of the cell cycle (Schiaffonati and Baserga, 1977), show highly organized actin cables.

Acknowledgements This work was supported by Grant No. 83-05-19 from the National Center for Nervous, Mental and Muscular Disorders (NCNMMD) of the Ministry of Health and Welfare, Japan.

\section{REFERENCES}

Azzarone, B., Mareel, M., Billard, P., Scemama, P., Chaponnier, C., and Macieira-Coelho, A. 1984. Abnormal properties of skin fibroblasts from patients with breast cancer. Int. J. Cancer 33: 759-764.

Barak, L.S., Nothnagel, E.A., DeMarco, E.F., and Webb, W.W. 1981. Differential staining of actin in metaphase spindle with 7-nitrobenz-2-oxa-1,3-diazole-phallacidin and fluorescent DNase: Is actin involved in chromosomal movement? Proc. Natl. Acad. Sci. U.S.A. 78; 3034-3038.

Kopelovitch, L. 1977. Phenotypic markers in human skin fibroblasts as possible diagnostic indices of hereditary adenomatosis of the colon and rectum. Cancer 40: 2534 2541.

Kopelovitch, L., Conlon, S., and Pollack, R. 1977. Defective organization of actin in cultured skin fibroblasts from patients with inherited adenocarcinoma. Proc. Natl. Acad. Sci. U.S.A. 74: 3019-3022.

Kopelovitch, L., Bias, N.E., and Helson, L. 1979. Tumor promotor alone induces neoplastic 
transformation of fibroblasts from humans genetically predisposed to cancer. Nature 282: 619-621.

Kopelovitch, L., Lipkin, M., Blattner, W.A., Fraumeni, J.F., Jr., Lynch, H.T., and Pollack, P.E. 1980. Organization of actin-containing cables in cultured skin fibroblasts from individuals at high risk of colon cancer. Int. J. Cancer 26: 301-307.

Kopelovitch, L. 1982. Genetic preposition to cancer in man: In vitro studies. Int. Rev. Cytol. 77 : 63-88.

Kossakowska, A.E., Gallie, B.L., and Phillip, R.A. 1982, Fibroblasts from retinoblastoma patients: Enhanced growth in fetal calf serum and a normal response to ionizing radiation. J. Cell. Physiol. 111 : 15-20.

Krone, W., Zorlein, S., and Mao, R. 1981. Cell culture studies on neurofibromatosis (von Recklinghausen). I. Comparative growth experiments with fibroblasts at high and low concentrations of fetal calf serum. Hum. Genet. 58: 188-193.

Mulvihill, J.J. 1977. Genetic repository of human neoplasia. In Genetics of Human Cancer, Mulvihill, J.J., Miller, R.W., and Fraumeni, J.F., Jr., eds., Raven Press, New York, pp. 137-143.

Ohno, K. and Kimura, G. 1984. Genetic analysis of control of proliferation in fibroblastic cells in culture. II. Alteration in proliferative and survival phenotypes in a set of temperature-sensitive mutants of rat $3 Y 1$ cells after infection or transformation with simian virus 40. Somat. Cell Mol. Genet. 10: 29-36.

Ohno, K. and Takeshita, K. 1984a. Patients with tuberous sclerosis have fibroblasts with normal limits for growth characteristics and sensitivities to DNA alkylating agents. Jpn. J. Human. Genet. 29: 359-369.

Ohno, K. and Takeshita, K. 1984b. Serum requirements of skin fibroblasts from patients with tuberous sclerosis. Jpn. J. Human. Genet. 29:234 (Abstract).

Pfeffer, L. M., Lipkin, M., Stutman, P., and Kopelovitch, L. 1976. Growth abnormalities in cultured human skin fibroblasts. J. Cell. Physiol. 89: 29-37.

Pfeffer, L.M. and Kopelovitch, L. 1977. Differential genetic susceptibility of cultured human skin fibroblasts to transformation by Kirsten murine sarcoma virus. Cell 10: 313-320.

Rasheed, S., Rhim, J.S., and Gardner, E.J. 1983. Inherited susceptibility to retrovirus-induced transformation of Gardner syndrome cells. Am. J. Hum. Genet. 35: 919-931.

Rhim, J.S., Huebner, R.J,, Arnstein, P., and Kopelovitch, L. 1980. Chemical transformation of cultured human skin fibroblasts derived from individuals with hereditary adenomatosis of the colon and rectum. Int. J. Cancer 26: 565-569.

Schiaffonati, L. and Baserga, R. 1977. Different survival of normal and transformed cells exposed to nutritional conditions nonpermissive for growth. Cancer Res. 37: 541-545.

Schneider, E.L. and Mitsui, Y. 1976. The relationship between in vitro cellular aging and in vivo human age. Proc. Natl. Acad. Sci. U.S.A. 73: 3584-3588.

Smith, J.R., Pereira-Smith, O.M., and Schneider, E.L. 1978. Colony size distributions as a measure of in vivo and in vitro aging. Proc. Natl. Acad. Sci. U.S.A. 75: 1353-1356.

Verderame, M., Alcorta, D., Engor, M., Smith, K., and Pollack, R. 1980. Cytoskeletal F-actin patterns quantitated with fuorescein isothiocyanate-phalloidin in normal and transformed cells. Proc. Natl. Acad. Sci. U.S.A. 77: 6624-6628.

Wang, E. and Gundersen, D. 1984. Increased organization of cytoskeleton accompanying the aging of human fibroblasts in vitro. Exp. Cell Res, 154: 191-202.

Weber, K., Lazarides, E., Goldman, R.D., Vogel, A., and Pollack, R. 1974. Localization and distribution of actin fibers in normal, transformed and revertant cells. Cold Spring Harbor Symp. Quant. Biol. 39: 363-369.

Yanishevsky, R., Mendelsohn, M.L., Mayall, B.H., and Cristofalo, V.J. 1974. Proliferative capacity and DNA content of aging human diploid cells in culture: A cytophotometric and autoradiographic analysis. J. Cell. Physiol. 82: 165-170. 